\title{
Prediction of SARS-CoV-2 Main Protease Inhibitors from Several Medicinal Plant Compounds by Drug Repurposing and Molecular Docking Approach.
}

Sayma Farabi*, Nihar Ranjan Saha, Noushin Anika Khan, Md. Hasanuzzaman.

Department of Biotechnology, Bangladesh Agricultural University, Mymensingh-2202, Bangladesh

*Correspondence:-E-mail: farabi.bge@gmail.com

\begin{abstract}
Coronaviruses are endemic in humans and infections normally mild, such as the common cold but cross-species transmission has produced some unusually virulent strains which now causing viral pneumonia and in serious cases even acute respiratory distress syndrome and death. SARS-CoV2 is the most threatening issue which leads the world to an uncertainty alongside thousands of regular death scenes. For this virus, death toll is increasing in. An effective vaccine to cure this virus is not yet available, thus requires concerted efforts at various scales. The viral Main Protease controls Coronavirus replication and is a proven drug discovery target for SARS-CoV-2. Here, comprehensive computational approaches including drug repurposing and molecular docking were employed to predict the efficacy of medicinal plant-based bioactive compounds against SARSCoV-2 Mpro. Molecular docking was performed using PyRx-autodock vina to analyze the inhibition probability. MPP (6LU7) was docked with 90 phytochemical compounds and docking was analysed by PyRx-autodock vina, Pymol version 1.7.4.5 Edu, and Biovia Discovery Studio 4.5. Furthermore, ADME analysis along with analysis of toxicity was also investigated to check the pharmacokinetics and drug-likeness properties of the antiviral phytochemicals. Remdesivir and lopinavir were used as standards for comparison. Our analyses revealed that the top ten (Azadirachtin, -12.5kcal $/ \mathrm{mol}$; Rutin, $-9 \mathrm{kcal} / \mathrm{mol}$; Theaflavin, $-9 \mathrm{kcal} / \mathrm{mol}$; Astragalin, -8.8 $\mathrm{kcal} / \mathrm{mol}$; Isoquercitrin, $-8.7 \mathrm{kcal} / \mathrm{mol}$; Hyperoside, $-8.6 \mathrm{kcal} / \mathrm{mol}$; Baicalin, $-8.4 \mathrm{kcal} / \mathrm{mol}$; Saponin, -8.3 kcal/mol; Sennoside A, -8.3 kcal/mol; Aloin, -8.2 kcal $/ \mathrm{mol}$, while Remdesivir and Lopinavir showed -8.2 and $-7.9 \mathrm{kcal} / \mathrm{mol}$ ) hits might serve as potential anti- SARS-CoV-2 lead molecules for further optimization and drug development process to combat COVID-19.
\end{abstract}

Keywords: SARS-CoV-2, Molecular Docking, Drug Repurposing, Medicinal Plant Compounds, Virtual screening, Drug design.

\section{Introduction}

The novel coronavirus is not a new virus to the world, a new strain of coronavirus family. The novel coronavirus was first reported on December 30, 2019 in Wuhan city, Hubei province, P.R. China [1]. The World Health Organization (WHO) temporarily named this pathogen 2019 novel coronavirus (2019-nCoV) and on February 12, 2020 finally released as the official name, SARSCoV-2 (severe acute respiratory syndrome coronavirus-2) [2]. The disease has the features like fever, cough and sever shortness of breathing, nausea, vomiting and diarrhea [3]. COVID-19 has not only created health problems but also created virtual boundaries that isolated corona infected 
country from rest of the world. This disease created medical emergency at global level, thus badly affected the international travelling, tourism and trade.

The first whole-genome sequence of SARS-CoV-2 was published on January 24, 2020 which helped the researchers to quickly screen the viral strain of this disease [4]. An article related to COVID-19 was first published on January 21 and from there we find that the virus belongs to the beta-coronavirus group, which share ancestry with bat coronavirus HKU9-1, similar to SARScoronaviruses and its spike protein interacts strongly with the human ACE2 receptor despite having sequence diversity [1]. On January 30, the WHO declared a Public Health Emergency of International Concern (PHEIC) for the 2019-nCoV outbreak. The first pandemic caused by a coronavirus, 213 countries has been reported with SARS-CoV-2 infection till 4 June 2020 causing 6,688,679 confirmed cases and 392,123 total deaths [5, 6]. Epidemiological data have determined person to person transmission as the route of the rapid outbreak of COVID-19, which has become a major obstruction in combating the virus $[7,8]$. Clinical studies reported that older patients have a higher case of fatality rate (CFR) than the young, and males have a higher CFR than female [8]. Apart from acute respiratory distress, COVID-19 patients have been diagnosed with higher rate of renal impairment, indicating the development of kidney dysfunction [9]. Unfortunately, there are no proven drugs, vaccines or therapies available to fight against COVID -19.

Computational biology and the molecular docking strategy have a variety of drug discovery applications, providing new insights on screening for potential drugs for the treatment of COVID19.These approaches also aid the understanding of the protein-ligand interactions as well as in figuring out drug surface hotspot, which are important for the discovery of an effective drug [10, 11]. In addition, drug repurposing approach is used for the identification of existing drugs for one disease, for the treatment of another disease. As the long-term solution, vaccine, will take years to be marketable, effective repurposing of existing drug remains the only alternative way to fight against an emerging disease like COVID-19 [12-15].

Currently, no specific therapies for COVID-19 are available and studies on the treatment of COVID-19 are lacking [16]. However, the measures that have been implemented remain limited to preventive and supportive therapies, designed to prevent further complications and organ damage [16]. Some preliminary studies have investigated potential combinations that include the protease inhibitor lopinavir/ritonavir, which is commonly used to treat human immunodeficiency virus (HIV)/acquired immunodeficiency syndrome patients, for the treatment of COVID-19infected patients [17]. Other reported antiviral treatments form human pathogenic CoVs include nucleoside analogues, neuraminidase inhibitors, remdesivir, umifenovir (arbidol), tenofovir disoproxil (TDF) and lamivudine (3TC) [17]. A separate investigation performed by Xu et al. (2020) indicated that among 4 tested drugs (nelfinavir, pitavastatin, perampanel, and praziquantel), nelfinavir was identified as the best potential inhibitor against COVID-19 Mpro, based on binding free energy calculations using the molecular mechanics with generalised Born and surface area solvation (MM/GBSA) model and solvated interaction energy (SIE) methods [19]. The results from preliminary studies remain unapproved for therapeutic use in clinical settings for the treatment of COVID-19-infected patients [18, 20]. Liu et al. (2020) have successfully crystallized the main protease (Mpro)/chymotrypsin-like protease (3CLpro) from COVID-19, which has been structured and repositioned in the Protein Data Bank (PDB) and is accessible by the public. This protease represents a potential target for the inhibition of $\mathrm{CoV}$ replication [18]. Herbal extracts from ethnomedic plants have been used since ancient times and are known for their antiviral properties 
and their more tolerable side effects. Therefore, nature-based pharmacotherapy may be a suitable alternative to treat this viral disease. An extensive library has been built using traditional herbal medicines found in various countries in South Asia, which have previously been studied for their antiviral activity against various viruses. [19, 20]. Therefore, this study was conducted to obtain a structural description of SARS-CoV-2 3CLpro, and detect potent natural anti-COVID-19 compounds as potent inhibitors of Mpro using molecular docking strategy.

\section{Materials and Method}

2.1 Retrieval of the structure and preparation of target:

The 3D structure of the Main Protease Proteins (MPP) essential for replication of SARS-Cov-2 essential for virus replication (PDB ID: 6LU) [21, 22], was collected from RCSB Protein Data Bank [23] (https://www.rcsb.org/), in .pdb format.

The 6LU7 protein contains two chains, A and B, which form a homodimer. Chain A was used for macromolecule preparation. The native ligand for 6LU7 is $\mathrm{n}$-[(5-methylisoxazol-3yl) carbonyl] alanyl-1-valyl-n 1 -((1r,2z)-4-(benzyloxy)-4-oxo-1-\{[(3r)-2-oxopyrrolidin-3yl]methyl\}but-2enyl)-l-leucinamide [21]. Before molecular docking, the 3D structure of MPP was retrieved from protein data bank (PDB ID: 6LU7) and was cleaned up by using Biovia Discovery Studio 4.5 [24] as it was in a complex structure with an inhibitor. After the removal of unwanted molecules such as water the protein was taken to dock with ligands.

\subsection{Ligand and Drug Scan}

A comprehensive library of medicinal plant containing phytochemicals with potential anti-viral activity and traditional medicinal compounds was produced from previously published studies (Supplementary Table 1) [25-42] and screened against the SARS-CoV-2 MPP. The 3-dimensional (3D) structure was obtained from PubChem (https://pubchem.ncbi.nlm.nih.gov/), in .sdf format.

\subsection{Determination of Active Sites}

The amino acids in the active site of a protein were determined using the Computed Atlas for Surface Topography of Proteins (CASTp) (http://sts.bioe.uic.edu/castp/index.html?201l) and Biovia Discovery Studio 4.5 [24]. The determination of the amino acids in the active site was used to analyze the Grid box and docking evaluation results.

\subsection{Molecular docking}

The PyRx [43] software of molecular docking approaches was employed for the screening of the drugs against Main Protease Protein of SARS-CoV-2. The protein was exposed to 90 phytochemicals for analyzing the highest negative binding energy and interactive amino acids. Recent drug repurposing studies proposed few drugs that target SARS-CoV-2 3CLpro and suggested that they could be used to treat COVID-19. Herein, we selected the best of these (Remdesivir and Lopinavir) from different drug repurposing studies [44] and docked them as 
controls in the present study. The grid box parameters were set to a size of $60 \mathrm{~A}^{\circ} \times 70 \mathrm{~A}^{\circ} \times 62 \mathrm{~A}^{\circ}$ $(\mathrm{x} \times \mathrm{y} \times \mathrm{z})$ and center of $-10.5011 \mathrm{~A}^{\circ} \times 13.9110 \mathrm{~A}^{\circ} \times 67.9200 \mathrm{~A}^{\circ}(\mathrm{x} \times \mathrm{y} \times \mathrm{z})$. LigPlot+ was used to generate the $2 \mathrm{D}$ ligand-protein interaction diagrams and to find out the involved amino acids with their interactive position in the docked molecules [45]. Discovery Studio and Pymol version 1.7.4.5 Edu were used to visualize and analyze the ligand molecules' interactions with the viral proteins [24].

\subsection{Drug Likeness Properties Analysis of the Screened Compounds}

To assess the absorption, distribution, metabolism, and excretion (ADME) properties of the topmost phytochemical candidates for the MPP inhibitors the Swiss ADME portal was used [46]. The study checked out the physico-chemical parameters (formula molecular weight, molar refractivity, TPSA), lipophilicity (Log Po/w (iLOGP), Log Po/w (XLOGP3), Log Po/w (WLOGP), Log Po/w (MLOGP), Log Po/w, (SILICOS-IT), Consensus Log Po/w), and water solubility (Log S: SILICOS-IT, solubility) of the screened topmost MPP inhibitors. To study the drug interaction with the cytochromes P450 (CYP) is one of the crucial in drug discovery that is why the screened MPP inhibitors were employed to study the inhibition effect of different CYP isoforms (CYP1A2 inhibitor, CYP2C19 inhibitor, CYP2C9 inhibitor, CYP2D6 inhibitor, CYP3A4 inhibitor). However, other relevant pharmacokinetic parameters, such as gastrointestinal (GI) absorption, BBB (blood-brain barrier) permeant and P-gp substrate, were also investigated for putative drug candidate of main protease proteins. [47]

\section{Results and Discussion}

\subsection{Screening of MPP inhibitors against the MPP of SARS-CoV-2}

SARS-CoV-2 is an $800 \mathrm{kDa}$ polypeptide that tends to be cleaved by different proteases that are indispensable for the viral replication. Hence, these proteases play important role in the replication of viral and non-structural protein formation. In short, these non-structural proteins have greater potential to be target site for the antiviral drug for the treatment of SARS-CoV-2 [48]. Information of main protease protein is represented in Table 1.

Table 1: Protein target structures and active site amino acids (Biovia Discovery Studio 4.5, 2019) and the native ligand structure

\begin{tabular}{|l|l|l|}
\hline PDB ID & Macromolecule & Active site \\
\hline 6LU7 & $\begin{array}{l}\text { THR-24, THR-25,THR-26, } \\
\text { HIS-41, MET-49, TYR-54, } \\
\text { PHE-140, LEU-141, ASN-142, } \\
\text { GLY-143, SER-144, CYS-145, } \\
\text { HIS-163, HIS-164, MET-165, } \\
\text { GLU-166, LEU-167, PRO-168, } \\
\text { HIS-172, ASP-187, ARG-188, } \\
\text { GLN-189, THR-190, ALA- } \\
\text { I91, GLN-192. }\end{array}$ \\
\hline
\end{tabular}


In our study, two approved drugs for MPP inhibitors- Remdesivir: ebola treatment [49], currently researched as a potential treatment to SARS-CoV2; Lopinavir: HIV protease inhibitor [50], were used as positive control for the screening of antiviral phytochemicals as potential drugs. All the listed phytochemicals and MPP inhibitors were employed for molecular docking by using PyRxvirtual screening tool and Biovia Discovery Studio 4.5 was utilized to predict the interaction between the mentioned ligands and the MPP of SARS-CoV-2 (PDB ID: 6LU7). List of phytochemicals that showed good result is represented (table 2).

Table 2: Summary of top ranked antiviral phytochemicals screened against SARS-CoV-2 MMP receptor binding site.

\begin{tabular}{|c|c|c|c|c|}
\hline $\begin{array}{l}\text { Pubchem } \\
\text { ID }\end{array}$ & Phytochemical name & $\begin{array}{l}\text { Molecular } \\
\text { formula }\end{array}$ & Source name & Reference \\
\hline 121304016 & Remdesivir & $\mathrm{C}_{27} \mathrm{H}_{35} \mathrm{~N}_{6} \mathrm{O}_{8} \mathrm{P}$ & \multirow{2}{*}{$\begin{array}{l}\text { Positive } \\
\text { Control }\end{array}$} & \\
\hline 92727 & Lopinavir & $\mathrm{C}_{37} \mathrm{H}_{48} \mathrm{~N}_{4} \mathrm{O}_{5}$ & & \\
\hline 5281303 & Azadirachtin & $\mathrm{C}_{35} \mathrm{H}_{44} \mathrm{O}_{16}$ & Azadirachta indica & 25 \\
\hline 5280805 & Rutin & $\mathrm{C}_{27} \mathrm{H}_{30} \mathrm{O}_{16}$ & Azadirachta indica & 26 \\
\hline 135403798 & Theaflavin & $\mathrm{C}_{29} \mathrm{H}_{24} \mathrm{O}_{12}$ & Camellia sinensis & 27 \\
\hline 5282102 & Astragalin & $\mathrm{C}_{21} \mathrm{H}_{20} \mathrm{O}_{11}$ & Nigella sativa & 28 \\
\hline 5280804 & Isoquercitrin & $\mathrm{C}_{21} \mathrm{H}_{20} \mathrm{O}_{12}$ & Camellia sinensis & 29 \\
\hline 5281643 & Hyperoside & $\mathrm{C}_{21} \mathrm{H}_{20} \mathrm{O}_{12}$ & Abelmoschus manihot & 30 \\
\hline 64982 & Baicalin & $\mathrm{C}_{21} \mathrm{H}_{18} \mathrm{O}_{11}$ & Scutellaria baicalensis & 31 \\
\hline 198016 & Saponin & $\mathrm{C}_{58} \mathrm{H}_{94} \mathrm{O}_{27}$ & Tieghemella heckelii & 32 \\
\hline 73111 & Sennoside A & $\mathrm{C}_{42} \mathrm{H}_{38} \mathrm{O}_{20}$ & Rheum palmatum & 33 \\
\hline 12305761 & Aloin & $\mathrm{C}_{21} \mathrm{H}_{22} \mathrm{O}_{9}$ & Aloe vera & 34 \\
\hline 65064 & $\begin{array}{l}\text { Epigallocatechin-3- } \\
\text { gallate }\end{array}$ & $\mathrm{C}_{22} \mathrm{H}_{18} \mathrm{O}_{11}$ & Camellia sinensis & 35 \\
\hline 442630 & Carpaine & $\mathrm{C}_{28} \mathrm{H}_{50} \mathrm{~N}_{2} \mathrm{O}_{4}$ & Carica papaya & 36 \\
\hline 442893 & Cusparine & $\mathrm{C}_{19} \mathrm{H}_{17} \mathrm{NO}_{3}$ & Galipea officinalis & 37 \\
\hline 162350 & Isovitexin & $\mathrm{C}_{21} \mathrm{H}_{20} \mathrm{O}_{10}$ & Alium sativum & 38 \\
\hline 10247670 & Piperitol & $\mathrm{C}_{20} \mathrm{H}_{20} \mathrm{O}_{6}$ & Piper betle & 39 \\
\hline 5280443 & Apigenin & $\mathrm{C}_{15} \mathrm{H}_{10} \mathrm{O}_{5}$ & Camellia sinensis & 40 \\
\hline 5280863 & Kaempferol & $\mathrm{C}_{15} \mathrm{H}_{10} \mathrm{O}_{6}$ & Alium sativum & 41 \\
\hline 1794427 & Chlorogenic acid & $\mathrm{C}_{16} \mathrm{H}_{18} \mathrm{O}_{9}$ & Camellia sinensis & 42 \\
\hline
\end{tabular}

The binding affinities obtained from the docking of 6LU7 with all 90 selected ligands are represented in the supplementary table 1 . Interaction and docking information of selected top 18 antiviral medicinal plant compounds with two established MPP inhibitors are enlisted in Table 3. Among these screened antiviral phytochemicals, Azadirachtin- extract found from medicinal plant Azadirachta indica showed highest negative binding affinity $-12.5(\mathrm{kcal} / \mathrm{mol})$ which was followed by Rutin, extract from Nigella sativa represented $-9.0(\mathrm{kcal} / \mathrm{mol})$ and Theaflavin from Alium sativum $-9.0(\mathrm{kcal} / \mathrm{mol})$. While two approved MPP inhibitors produced -8.2 and $-7.9(\mathrm{kcal} / \mathrm{mol})$ respectively in our study. 
Table 3: Molecular docking results with interactive amino acids from SARS-CoV-2 MPP of top phytochemicals and approved MPP inhibitors.

\begin{tabular}{|c|c|c|c|c|}
\hline $\begin{array}{l}\text { No } \\
\text {. }\end{array}$ & $\begin{array}{l}\text { Phytochemical } \\
\text { name }\end{array}$ & $\begin{array}{l}\text { Molecular structure } \\
\text { and interaction with } \\
\text { 6LU7 }\end{array}$ & $\begin{array}{l}\text { Binding } \\
\text { Energy } \\
(\mathrm{kcal} / \mathrm{mol}) \\
\end{array}$ & $\begin{array}{l}\text { Involved amino acids and } \\
\text { positions }\end{array}$ \\
\hline 1 & Remdesivir & 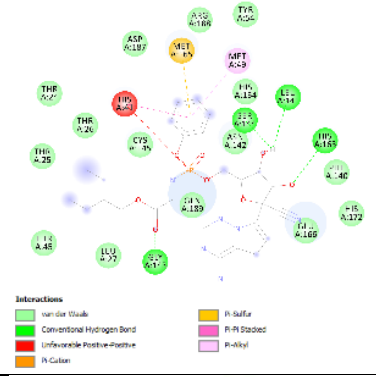 & -8.2 & $\begin{array}{l}\text { THR-24, THR-25,THR-26,LEU- } \\
\text { 27,THR-45, HIS-41, MET-49, } \\
\text { TYR-54, PHE-140, LEU-141, } \\
\text { ASN-142, GLY-143, SER-144, } \\
\text { CYS-145, HIS-163, HIS-164, } \\
\text { MET-165, GLU-166, HIS-172, } \\
\text { ASP-187, ARG-188, GLN-189, }\end{array}$ \\
\hline 2 & Lopinavir & 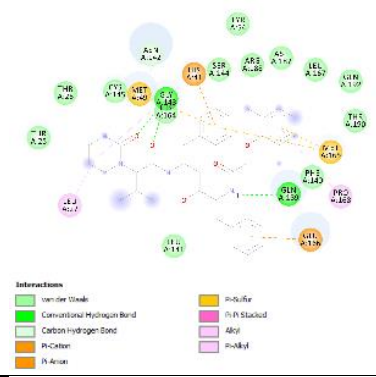 & -7.9 & $\begin{array}{l}\text { THR-26, THR-25, LEU-27, CYS- } \\
\text { 145, MET-49, ASN-142, GLY- } \\
\text { 143, HIS-164, HIS-41, TYR-54, } \\
\text { SER-144, ARG-188, } \\
\text { ASP-187, LEU-167, GLN-192, } \\
\text { THR-190, MET-165, PHE-140, } \\
\text { PRO-168, GLU-166, GLN-189, } \\
\text { LEU-141 }\end{array}$ \\
\hline 3 & Azadirachtin & 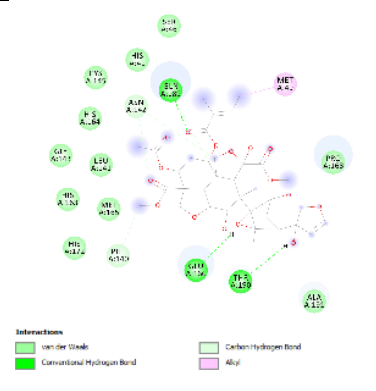 & -12.5 & $\begin{array}{l}\text { HIS-41,SER-46 MET-49, PHE- } \\
\text { 140, LEU-141, ASN-142, GLY- } \\
\text { 143, CYS-145, HIS-163, HIS- } \\
\text { 164, MET-165, GLU-166, PRO- } \\
\text { 168, HIS-172, GLN-189, THR- } \\
\text { 190, ALA-191, }\end{array}$ \\
\hline 4 & Rutin & 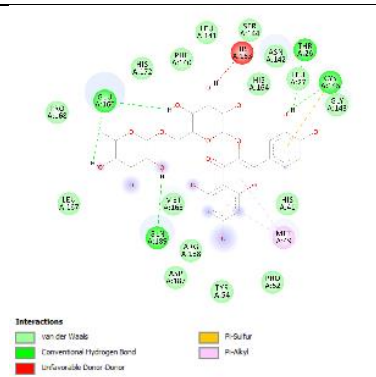 & -9.0 & $\begin{array}{l}\text { THR-26, LEU-27, HIS-41, MET- } \\
\text { 49, PRO-52, TYR-54, PHE-140, } \\
\text { LEU-141, ASN-142, GLY-143, } \\
\text { SER-144, CYS-145, HIS-163, } \\
\text { HIS-164, MET-165, GLU-166, } \\
\text { LEU-167, PRO-168, HIS-172, } \\
\text { ASP-187, ARG-188, GLN-189 }\end{array}$ \\
\hline 5 & Theaflavin & 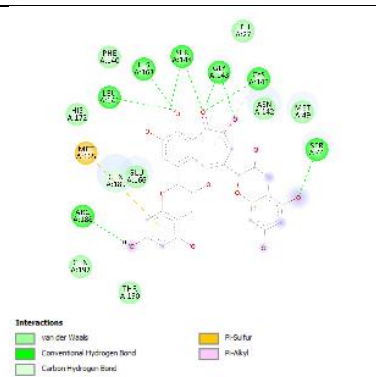 & -9.0 & $\begin{array}{l}\text { LEU-27, SER- 46, MET-49, } \\
\text { PHE-140, LEU-141, ASN-142, } \\
\text { GLY-143, SER-144, CYS-145, } \\
\text { HIS-163, MER-165, GLU-166, } \\
\text { HIS-172, ARG-188, GLN-189, } \\
\text { THR-190, GLN-192 }\end{array}$ \\
\hline
\end{tabular}




\begin{tabular}{|c|c|c|c|c|}
\hline 6 & Astragalin & 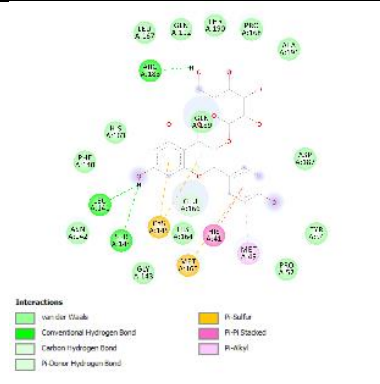 & -8.8 & $\begin{array}{l}\text { HIS-41, MET-49, PRO-52, TYR- } \\
\text { 54, PHE-140, LEU-141, ASN - } \\
\text { 142, GLY-143, SER-144, CYS- } \\
\text { 145, HIS-163, HIS-164, MET- } \\
\text { 165, GLU-166, LEU-167, PRO- } \\
\text { 168, ASP-187, ARG-188, GLN- } \\
\text { 189, THR-190, ALA-191, GLN- } \\
\text { 192. }\end{array}$ \\
\hline 7 & Isoquercitrin & 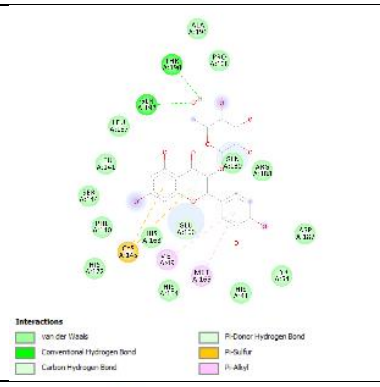 & -8.7 & $\begin{array}{l}\text { MET-41, MET-49, TYR-54, } \\
\text { PHE-140, LEU-141, SER-144, } \\
\text { CYS-154, HIS-163, HIS-164, } \\
\text { MET-165, GLU-166,LEU-167, } \\
\text { PRO-168, HIS-172, ASP-187, } \\
\text { ARG-188, GLN-189, THR-190, } \\
\text { ALA-191, GLN-192 }\end{array}$ \\
\hline 8 & Hyperoside & (2) & -8.6 & $\begin{array}{l}\text { HIS-41, MET-49, PRO-52, TYR- } \\
\text { 54, PHE-140, LEU-141, ASN- } \\
\text { 142, SER-144, CYS-145, HIS- } \\
\text { 163, HIS-164, MET-165, GLU- } \\
\text { 166, LEU-167, PRO-168, ASP- } \\
\text { 187, ARG-188, GLN-189, THR- } \\
\text { 190, GLN-192 }\end{array}$ \\
\hline 9 & Baicalin & 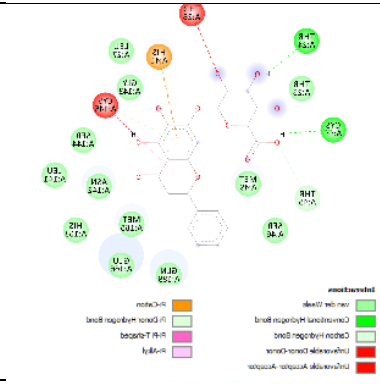 & -8.4 & $\begin{array}{l}\text { THR-25, THR-26, THR-27, } \\
\text { LEU-27, HIS-41, CYS-44, THR- } \\
\text { 45, SER-46, MET-49, LEU-141, } \\
\text { ASN-142, GLY-143, SER-144, } \\
\text { CYS-145, HIS-163, MET-165, } \\
\text { GLU-166, GLN-189. }\end{array}$ \\
\hline 10 & Saponin & 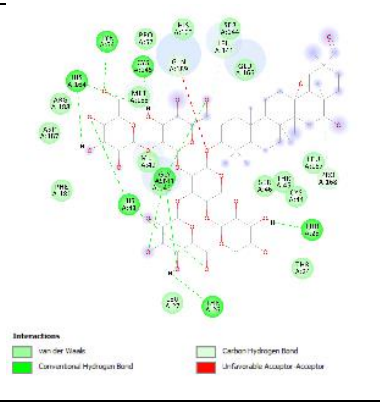 & -8.3 & $\begin{array}{l}\text { THR-24, THR-25, THR-26, } \\
\text { LEU-27, HIS-41, CYS-44, THR- } \\
\text { 45, SER-46, MET-49, PRO-52, } \\
\text { TYR-54, LEU-141, ASN-142, } \\
\text { GLY-143, SER-144, CYS-145, } \\
\text { HIS-163, HIS-164, MET-165, } \\
\text { GLU-166, LEU-167, PRO-168, } \\
\text { PHE-181, ASP-187, ARG-188, } \\
\text { GLN-189 }\end{array}$ \\
\hline
\end{tabular}




\begin{tabular}{|c|c|c|c|c|}
\hline 11 & Sennoside A & 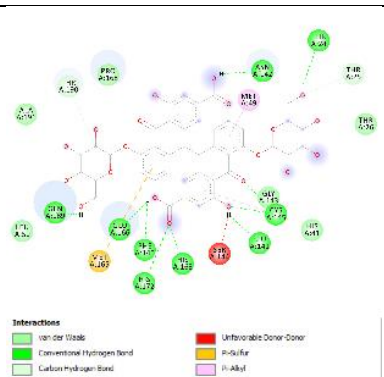 & -8.3 & $\begin{array}{l}\text { THR-24, THR-25, THR-26, HIS- } \\
\text { 41, MET-49, LEU-50, PHE-140, } \\
\text { LEU-141, ASN-142, GLY-143, } \\
\text { SER-144, CYS-145, HIS-163, } \\
\text { GLU-166, PRO-168, HIS-172, } \\
\text { GLN-189, THR-190, ALA-191 }\end{array}$ \\
\hline 12 & Aloin & 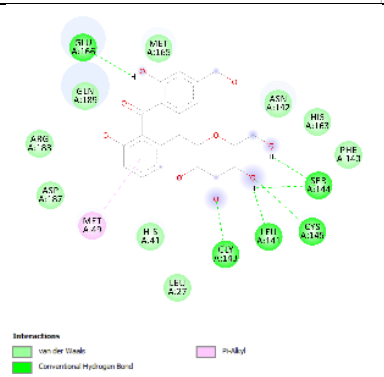 & -8.2 & $\begin{array}{l}\text { LEU-27, HIS-41, MET-49, PHE- } \\
\text { 140, LEU-141, ASN-142, GLY- } \\
\text { 143, SER-144, CYS-145, HIS- } \\
\text { 163, MET-165, GLU-166, ASP- } \\
\text { 187, ARG-188, GLN-189. }\end{array}$ \\
\hline 13 & Isovitexin & 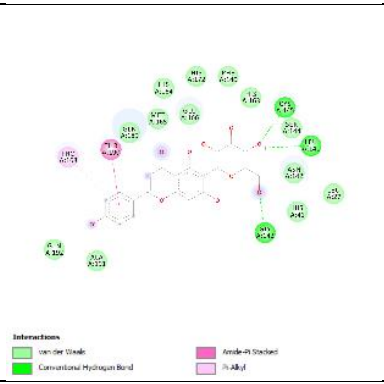 & -8.0 & $\begin{array}{l}\text { LEU-27, HIS-41, PHE-140, LEU- } \\
\text { 141, ASN-142, GLY-143, SER- } \\
\text { 144, CYS-145, HIS-163, HIS- } \\
\text { 164, MET-165, GLU-166, PRO- } \\
\text { 188, HIS-172, GLN-189, THR- } \\
\text { 190, ALA-191, GLN-192 }\end{array}$ \\
\hline 14 & $\begin{array}{l}\text { Epigallocatechin } \\
\text {-3-gallate }\end{array}$ & 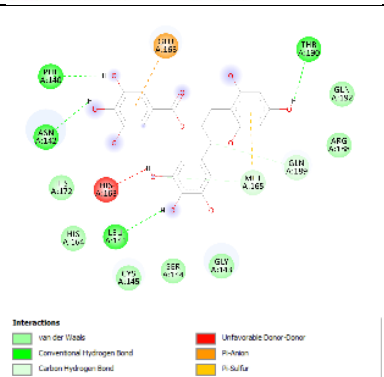 & -7.9 & 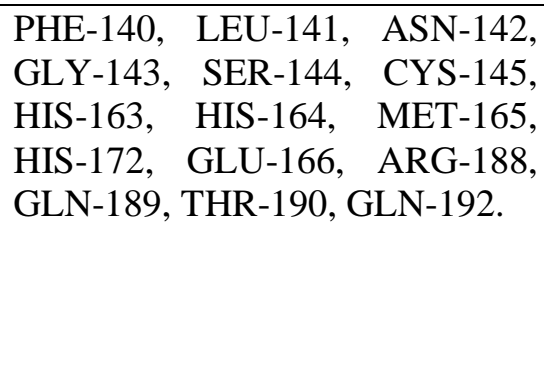 \\
\hline 15 & Carpaine & ats & -7.9 & $\begin{array}{l}\text { MET-165, GLU-166, PRO-168, } \\
\text { GLN-189, THR-190 }\end{array}$ \\
\hline
\end{tabular}




\begin{tabular}{|c|c|c|c|c|}
\hline 16 & Cusparine & 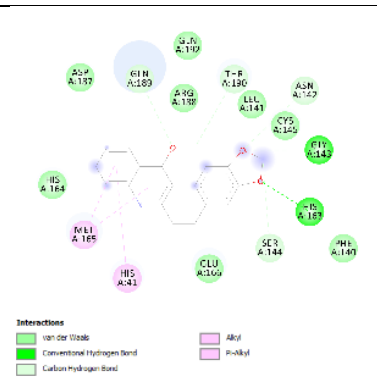 & -7.9 & $\begin{array}{lcc}\text { HIS-41, } & \text { PHE-140, } & \text { LEU-141, } \\
\text { ASN-142, } & \text { GLY-143, } & \text { SER-144, } \\
\text { CYS-145, } & \text { HIS-163, } & \text { HIS-164, } \\
\text { MET-165, } & \text { GLU-166, } & \text { ASP-187, } \\
\text { ARG-188, } & \text { GLN-189, } & \text { THR-190, } \\
\text { GLN-192. } & \end{array}$ \\
\hline 17 & Piperitol & 政 & -7.8 & $\begin{array}{l}\text { PHE-140, LEU-141, ASN-142, } \\
\text { GLY-143, SER-144, CYS-145, } \\
\text { HIS-163, MET-165, GLU-166, } \\
\text { PRO-168, HIS-172, GLN-189, } \\
\text { THR-190, ALA-191 }\end{array}$ \\
\hline 18 & Apigenin & 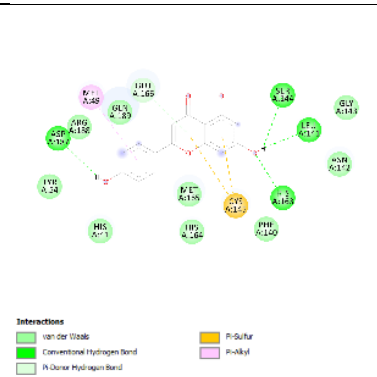 & -7.8 & $\begin{array}{l}\text { HIS-41, MET-49, TYR-54, PHE- } \\
\text { 140, LEU-141, ASN-142, GLY- } \\
\text { 143, SER-144, CYS-145, HIS- } \\
\text { 163, HIS-164, MET-165, GLU- } \\
\text { 166, ASP-187, ARG-188, GLN- } \\
\text { 189. }\end{array}$ \\
\hline 19 & Kaempferol & 에 & -7.8 & $\begin{array}{l}\text { HIS-41, MET-49, TYR-54, PHE- } \\
\text { 140, LEU-141, ASN-142, GLY- } \\
\text { 143, SER-144, CYS-145, HIS- } \\
\text { 163, HIS-164, MET165, GLU- } \\
\text { 166, ASP-187, ARG-188, GLN- } \\
189\end{array}$ \\
\hline 20 & Chlorogenic acid & (1ii) & -7.8 & $\begin{array}{l}\text { THR-25, THR-26, LEU-27, HIS- } \\
\text { 41, MET-49, PHE-140, LEU-141, } \\
\text { ASN-142, GLY-143, SER-144, } \\
\text { CYS-145, HIS-163, HIS-164, } \\
\text { MET-165, GLU-166, HIS-172, } \\
\text { GLN-189. }\end{array}$ \\
\hline
\end{tabular}

Here, Remdesivir was found to be involved with the amino acid of Thr-24, Thr-25,Thr-26,Leu27, Thr-45, His-41, Met-49, Tyr-54, Phe-140, Leu-141, Asn-142, Gly-143, Ser-144, Cys-145, His163, His-164, Met-165, Glu-166, His-172, Asp-187, Arg-188, Gln-189 in the MPP (PDB ID 6LU7) of SARS-CoV-2 (Figure 1). Azadirachtin exhibited the highest binding energy at the active 
site of COVID-19 and it formed interactions with His-41,Ser-46 Met-49, Phe-140, Leu-141, Asn142, Gly-143, Cys-145, His-163, His-164, Met-165, Glu-166, Pro-168, His-172, Gln-189, Thr190, Ala-191 (Figure 2a, Table: 3). Results of this study shown that Thr-26, Leu-27, His-41, Met49, Pro-52, Tyr-54, Phe-140, Leu-141, Asn-142, Gly-143, Ser-144, Cys-145, His-163, His-164, Met-165, Glu-166, Leu-167, Pro-168, His-172, Asp-187, Arg-188, Gln-189 were critical residues for the binding of Rutin to protease protein (Figure 2b, Table 3). Active site residues Leu-27, Ser46, Met-49, Phe-140, Leu-141, Asn-142, Gly-143, Ser-144, Cys-145, His-163, Mer-165, Glu-166, His-172, Arg-188, Gln-189, Thr-190, Gln-192 participated in interactions with Theaflavin (Figure 2c, Table 3).

\subsection{Drug Likeness Properties Analysis of the Screened phytochemicals}

The physico-chemistry, pharmacokinetics, medicinal chemistry friendliness and toxicity of top ten phytochemicals found by docking were analyzed by SwissADME. The physiochemical parameters, lipophilicity and water solubility of these compounds are described in Table 4. Water solubility was also studied in this study and found that all the compounds were moderately soluble. Other important properties such as Molecular weight (MW), molecular refractivity (MR) and topological polar surface area (TPSA), which are very useful for the estimation of ADME properties were also included in this study.

Remarkably, none of the screened compounds showed any undesired effects such as mutagenicity, tumorigenicity, irritating and reproductive effects. However, these compounds showed CYP450 enzymes inhibition effects except CYP1A2. However, GI absorption was found low in case of every phytochemicals. The blood-brain barrier (BBB) permeation was also calculated by BOILED-Egg models [30], and there was no BBB permeant among putative MPP inhibitors. The study revealed the water solubility levels of Azadirachtin $(3.33 \mathrm{e}-02 \mathrm{mg} / \mathrm{ml} ; 4.62 \mathrm{e}-05 \mathrm{~mol} / \mathrm{l})$, Rutin (3.08e-01 mg/ml ; 5.05e-04 mol/l), Theaflavin $(3.39 \mathrm{e}-02 \mathrm{mg} / \mathrm{ml} ; 6.01 \mathrm{e}-05 \mathrm{~mol} / \mathrm{l})$, Astragalin $(3.55 \mathrm{e}+00 \mathrm{mg} / \mathrm{ml} ; 7.91 \mathrm{e}-03 \mathrm{~mol} / \mathrm{l})$, Isoquercitrin $(1.43 \mathrm{e}+01 \mathrm{mg} / \mathrm{ml} ; 3.08 \mathrm{e}-02 \mathrm{~mol} / \mathrm{l})$, Hyperoside $(1.43 \mathrm{e}+01 \mathrm{mg} / \mathrm{ml} ; 3.08 \mathrm{e}-02 \mathrm{~mol} / \mathrm{l})$, Baicalin $(2.67 \mathrm{e}+00 \mathrm{mg} / \mathrm{ml} ; 5.98 \mathrm{e}-03 \mathrm{~mol} / \mathrm{l})$, Saponin $(6.09 \mathrm{e}+05 \mathrm{mg} / \mathrm{ml} ; 4.98 \mathrm{e}+02 \mathrm{~mol} / \mathrm{l})$, Sennoside A $(1.07 \mathrm{e}+00 \mathrm{mg} / \mathrm{ml} ; 1.24 \mathrm{e}-03 \mathrm{~mol} / \mathrm{l})$, Aloin $(6.77 \mathrm{e}+00 \mathrm{mg} / \mathrm{ml} ; 1.62 \mathrm{e}-02 \mathrm{~mol} / \mathrm{l})$. 
Table 4: ADME analysis of top ten MPP phytochemical inhibitors by using SwissADME

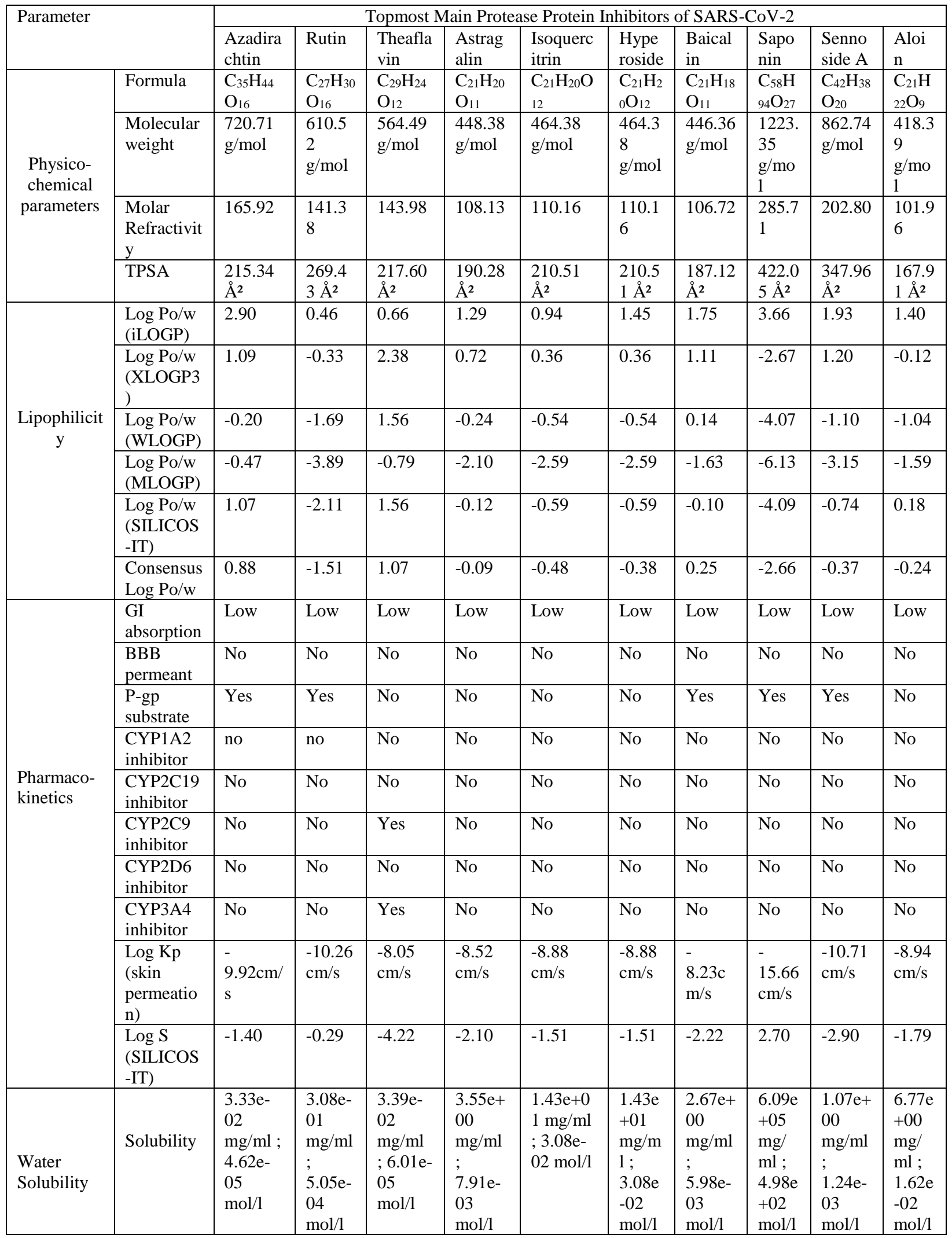



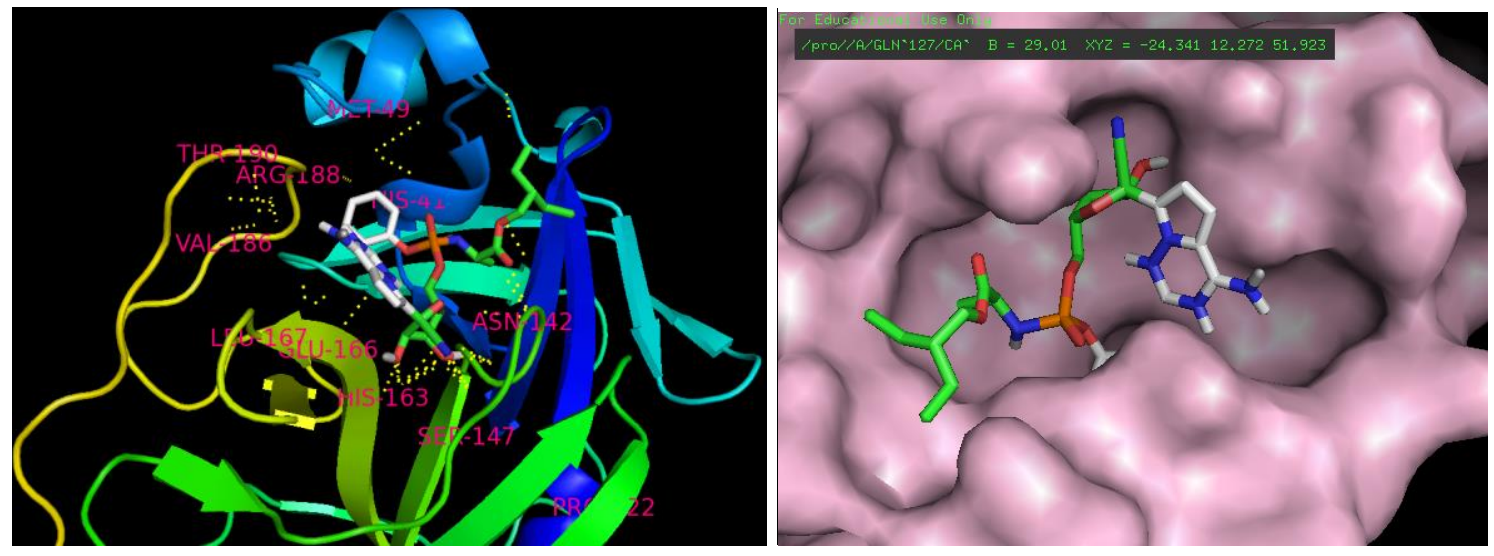

Figure 1: Molecular interaction between Remdesivir and Main Protease Protein of SARS-CoV-2.

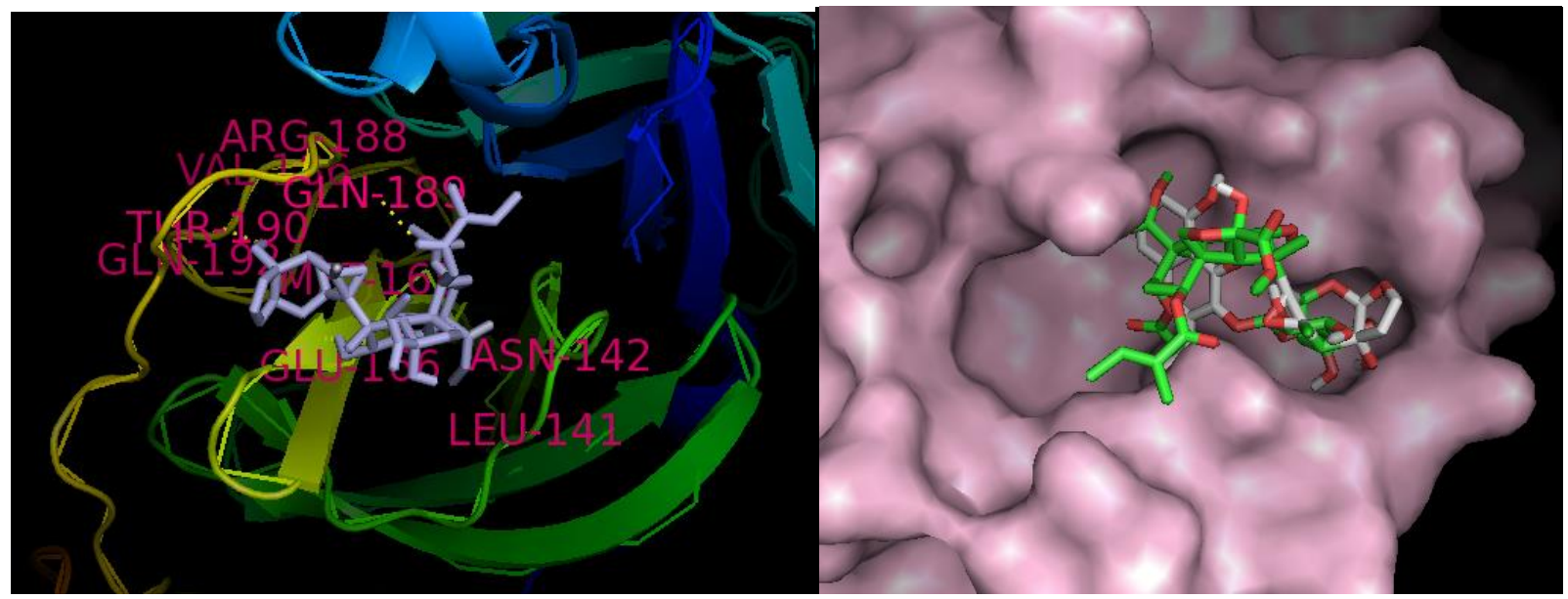

(2a)

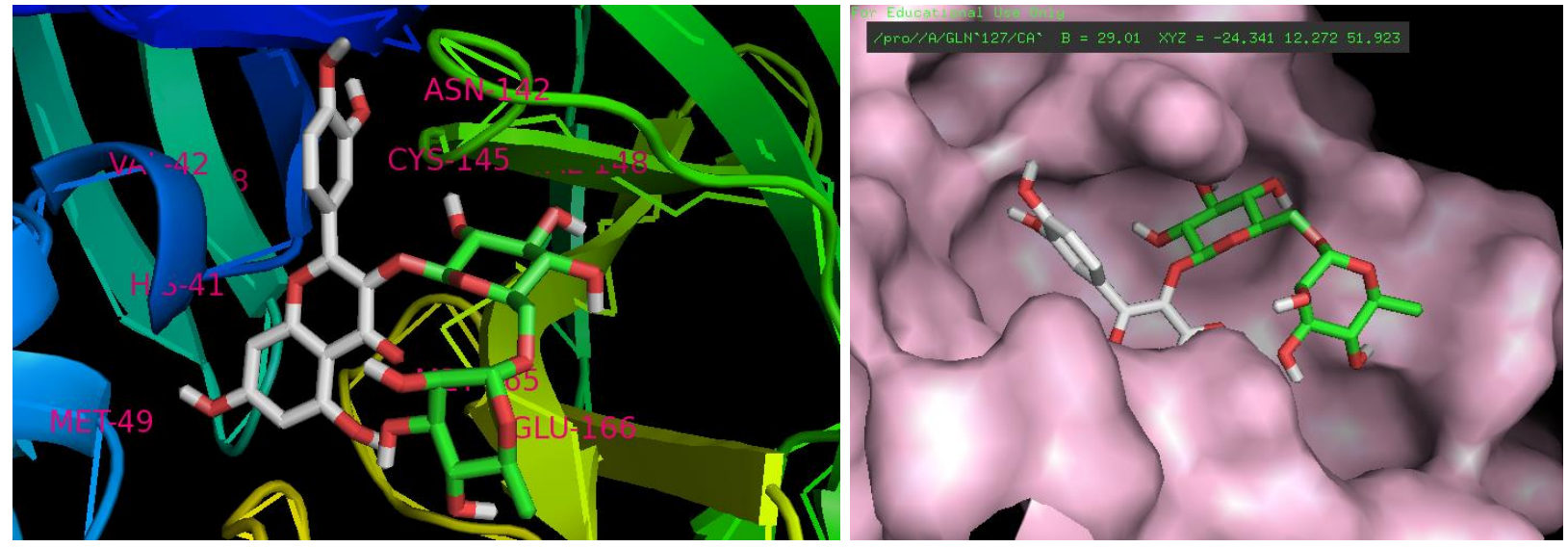

2 (b) 

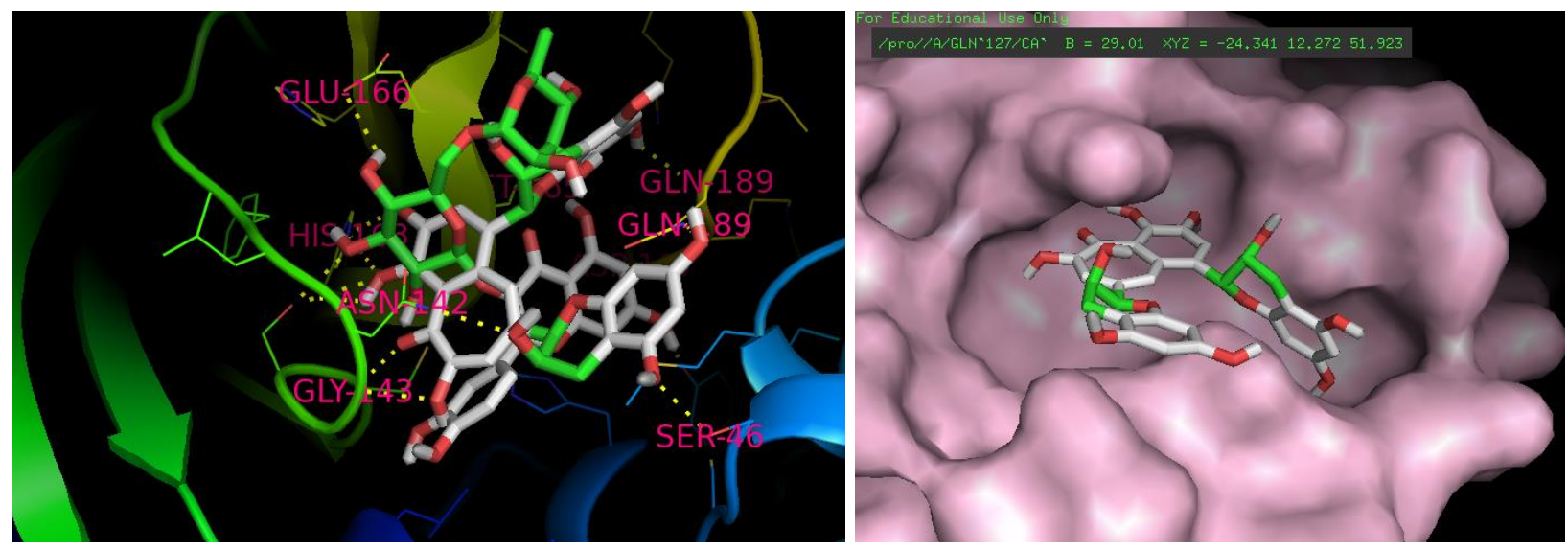

2(c)

Figure 2: Molecular insights of Main Protease Protein interactions with (a) Azadirachtin (12.5 kcal/mol) (b) Rutin $(9.0 \mathrm{kcal} / \mathrm{mol})$ (c) Theaflavin $(9.0 \mathrm{kcal} / \mathrm{mol})$

\section{Conclusions}

COVID-19 has created a catastrophic global crisis affecting thousands of people every day, having already claimed thousands of lives, and severely hampered the global economy. To contribute this fight against COVID-19, virtual screening based molecular docking was carried out to identify new compounds which could bind the Mpro of COVID-19. Our study propose that phytochemicals such as Azadirachtin, Rutin, Theaflavin, Astraglin, Isoquercitrin, Hyperoside, Baicalin, Saponin, Sennoside-A have a better binding affinity to Mpro of COVID-19 than Remdesivir and Lopinavir. Further in-vitro and in-vivo analyses are required to transform these potential inhibitors into clinical drugs. We anticipate that the insights obtained in the present study may prove valuable for exploring and developing novel natural anti-COVID-19 therapeutic agents in the future. 
Supplementary Table 1: Sources and Molecular Docking results of all the phytochemicals studied as antiviral agent

\begin{tabular}{|c|c|c|c|c|c|}
\hline Phytochemical name & Source name & $\begin{array}{c}\text { Binding } \\
\text { energy } \\
(\mathrm{kcal} / \mathrm{mol})\end{array}$ & Phytochemical name & Source name & $\begin{array}{c}\text { Binding } \\
\text { energy } \\
(\mathrm{kcal} / \mathrm{mol})\end{array}$ \\
\hline $\begin{array}{l}\text { Epigallocatechin-3- } \\
\text { gallate }\end{array}$ & Camellia sinensis & -7.9 & Gallocatechin & Camellia sinensis & -7.2 \\
\hline Curcumin & Curcuma longa & -7.1 & Epiafzelechin & Camellia sinensis & -7 \\
\hline Baicalin & Scutellaria baicalensis & -8.4 & Epicatechin & Camellia sinensis & -7 \\
\hline Azadirachtin & Azadirachta indica & -12.5 & Epigallocatechin & Camellia sinensis & -7.1 \\
\hline Nimbin & Azadirachta indica & -7 & Linalool & Camellia sinensis & -4.7 \\
\hline Dammarenolic acid & Azadirachta indica & -6.4 & $\begin{array}{c}1,2,4 \text {-trihydroxy- } \\
\text { benzene }\end{array}$ & Camellia sinensis & -4.9 \\
\hline Excoecarianin & Azadirachta indica & -3.4 & 4-terpineol & Camellia sinensis & -4.7 \\
\hline Honokiol & Azadirachta indica & -6.5 & Allantoin & Camellia sinensis & -5.4 \\
\hline Oleanane & Azadirachta indica & -7.3 & Alpha-amyrin & Camellia sinensis & -7.7 \\
\hline Quercetin & Azadirachta indica & -7.3 & Alpha-pinene & Camellia sinensis & -4.3 \\
\hline Saikosaponins & Azadirachta indica & -5.5 & Alpha-terpineol & Camellia sinensis & -4.8 \\
\hline Sennoside a & Rheum palmatum & -8.3 & Apigenin & Camellia sinensis & -7.8 \\
\hline Silvestrol & Azadirachta indica & -7.1 & Aromadendrin & Camellia sinensis & -7.6 \\
\hline Sjp-1-5 & Azadirachta indica & -6.3 & $\begin{array}{c}\text { Beta- } \\
\text { sesquiphellandrene }\end{array}$ & Camellia sinensis & -5.9 \\
\hline Xanthohumol & Azadirachta indica & -7.7 & Caffeic-acid & Camellia sinensis & -5.7 \\
\hline 3-methylquercetin & Azadirachta indica & -7.3 & Caffeine & Camellia sinensis & -5.2 \\
\hline Saponin & Tieghemella heckelii & -7.9 & Campesterol & Camellia sinensis & -6.9 \\
\hline Gingerol & Tieghemella heckelii & -6 & Carvacrol & Camellia sinensis & -4.8 \\
\hline Lutein & Spinach & -6.5 & Chlorogenic-acid & Camellia sinensis & -7.8 \\
\hline Pyrrolidine & Raphanus sativus & -3.1 & Dammaradienol & Camellia sinensis & -6.9 \\
\hline Sinapine & Raphanus sativus & -6.3 & Cryptoxanthin & Camellia sinensis & -6.6 \\
\hline Chavibetol & Piper betle & -4.9 & Diphenylamine & Camellia sinensis & -5.6 \\
\hline Eugenol & Piper betle & -5 & Eugenol & Alium sativum & -4.9 \\
\hline Piperitol & Piper betle & -7.8 & Farnesol & Alium sativum & -5.1 \\
\hline Carpaine & Carica papaya & -7.9 & Hyperoside & Alium sativum & -8.6 \\
\hline Nicotine & Nicotiana tabacum & -4.6 & Isoquercitrin & Alium sativum & -8.7 \\
\hline Reserpine & Rauwolfia serpentina & -7.3 & Isovitexin & Alium sativum & -7.9 \\
\hline 1,8-cineole & Citrus aurantiifolia & -4.2 & Kaempferol & Alium sativum & -7.8 \\
\hline Alpha-pinene & Citrus aurantiifolia & -3.9 & Lupeol & Alium sativum & -7.3 \\
\hline Cusparine & Galipea officinalis & -7.9 & Naringenin & Alium sativum & -7.7 \\
\hline Carvone & Nigella sativa & -4.7 & Theaflavin & Alium sativum & -9 \\
\hline Astragalin & Nigella sativa & -8.8 & Ajoene & Alium sativum & -4.2 \\
\hline Nigellicine & Nigella sativa & -6.9 & Allicin & Alium sativum & -3.3 \\
\hline Nigellidine & Nigella sativa & -7.6 & Allitridin & Allium sativum & -4.4 \\
\hline Rutin & Nigella sativa & -9 & Tinosporinone & Tinospora cordifolia & -5.1 \\
\hline Nigellone & Nigella sativa & -6.5 & syringin & Tinospora cordifolia & -3.6 \\
\hline Stigmasterol & Nigella sativa & -7.1 & Andrographolide & $\begin{array}{c}\text { Andrographis } \\
\text { paniculata }\end{array}$ & -6.9 \\
\hline Thymoquinone & Nigella sativa & -4.9 & Coumarin & Lico rice & 7 \\
\hline
\end{tabular}




\begin{tabular}{|c|c|c|c|c|c|}
\cline { 4 - 6 } Thymohydroquinone & Nigella sativa & -5 & Acemannan & Aloe vera & -5.4 \\
\hline Hederagenin & Nigella sativa & -6.9 & Aloe-emodin & Aloe vera & -7 \\
\hline Cycloartenol & Nigella sativa & -6.9 & Aloin & Aloe vera & -8.2 \\
\hline Citral & Abelmoschus esculentus & -4.4 & Lupeol & Aloe vera & -7.3 \\
\hline Gossypol & Abelmoschus esculentus & -7.2 & Rhein & Aloe vera & -7.5 \\
\hline Phylloquinone & Abelmoschus esculentus & -5.5 & Betulin & $\begin{array}{c}\text { Rhizophora } \\
\text { mucronata }\end{array}$ & -7.2 \\
\hline Cianidanol & Camellia sinensis & -7.1 & Gallocatechin & Camellia sinensis & -7.2 \\
\hline
\end{tabular}

\section{Reference}

[1] X. Xu, P. Chen, J. Wang, et al., Evolution of the novel coronavirus from the ongoing Wuhan outbreak and modeling of its spike protein for risk of human transmission, Sci. China Life Sci. 63 (2020) 457-460. Doi: 10.1007/s11427-020-1637-5

[2] Imai, N., Cori, A., Dorigatti, I., Baguelin, M., Donnelly, C. A., Riley, S., \& Ferguson, N. M. (2020). Report 3: Transmissibility of 2019-nCoV. online preprint.

[3] R. Porcheddu, C. Serra, D. Kelvin, N. Kelvin, S. Rubino, Similarity in Case Fatality Rates (CFR) of COVID-19/SARS-COV-2 in Italy and China, J. Infect. Dev. Ctries. 14 (2020) 125-128. Doi:10.3855/jidc. 12600.

[4] Zhu, N., Zhang, D., Wang, W., Li, X., Yang, B., Song, J., Zhao, X., Huang, B., Shi, W., \& Lu, R. (2020). A novel coronavirus from patients with pneumonia in China, 2019. New England Journal of Medicine. Doi: 10.1056/NEJMoa2001017

[5] Worldometer, Coronavirus Cases, Worldometer. (2020) 1-22. Doi:10.1101/2020.01.23.20018549V2.

[6] WHO, Coronavirus disease (COVID-19) Situation Dashboard, (n.d.). https://www.worldometers.info/coronavirus.

[7] N. Chen, M. Zhou, X. Dong, J. Qu, F. Gong, Y. Han, Y. Qiu, J. Wang, Y. Liu, Y. Wei, J. Xia, T. Yu, X. Zhang, L. Zhang, Epidemiological and clinical characteristics of 99 cases of 2019 novel coronavirus pneumonia in Wuhan, China: a descriptive study, Lancet. 395 (2020) 507-513. Doi:10.1016/S0140-6736(20)30211-7.

[8] Y. Yang, Q. Lu, M. Liu, Y. Wang, A. Zhang, N. Jalali, N. Dean, I. Longini, M.E. Halloran, B. $\mathrm{Xu}, \mathrm{X}$. Zhang, L. Wang, W. Liu, L. Fang, Epidemiological and clinical features of the 2019 novel coronavirus outbreak in China, MedRxiv. (2020) Doi:10.1101/2020.02.10.20021675.

[9] A.-2019-nCoV Volunteers, Z. Li, M. Wu, J. Guo, J. Yao, X. Liao, S. Song, M. Han, J. Li, G. Duan, Y. Zhou, X. Wu, Z. Zhou, T. Wang, M. Hu, X. Chen, Y. Fu, C. Lei, H. Dong, Y. Zhou, H. 
Jia, X. Chen, J. Yan, Caution on Kidney Dysfunctions of 2019-nCoV Patients, MedRxiv. (2020) 2020.02.08.20021212. Doi:10.1101/2020.02.08.20021212.

[10] S. Ekins, J. Mestres, B. Testa, In silico pharmacology for drug discovery: Methods for virtual ligand screening and profiling, Br. J. Pharmacol. 152 (2007) 9-20. Doi:10.1038/sj.bjp.0707305.

[11] R.E. Hubbard, I. Chen, B. Davis, Informatics and modeling challenges in fragment-based drug discovery, Curr. Opin. Drug Discov. Dev. 10 (2007) 289-297.

[12] A. Pizzorno, B. Padey, O. Terrier, M. Rosa-Calatrava, Drug repurposing approaches for the treatment of influenza viral infection: Reviving old drugs to fight against a long-lived enemy, Front. Immunol. 10 (2019) 531. Doi:10.3389/fimmu.2019.00531.

[13] B. Mercorelli, G. Palù, A. Loregian, Drug Repurposing for Viral Infectious Diseases: How Far Are We?, Trends Microbiol. 26 (2018) 865-876. Doi:10.1016/j.tim.2018.04.004.

[14] X. Li, J. Yu, Z. Zhang, J. Ren, A.E. Peluffo, W. Zhang, Y. Zhao, K. Yan, D. Cohen, W. Wang, Network bioinformatics analysis provides insight into drug repurposing for COVID-2019, (2020). Doi:10.20944/preprints202003.0286.v1.

[15] NCBI Resource Coordinators, Database resources of the National Center for Biotechnology Information., Nucleic Acids Res. 44 (2016) D7-D19. Doi:10.1093/nar/gkv1290.

[16] P. Rodríguez-Morales, Alfonso J; MacGregor, Kirsten; Kanagarajah, Sanch; Patel, Dipti; Schlagenhauf, "Going global - Travel and the 2019 novel coronavirus," Travel Med. Infect. Dis., vol. 33, 2020, Doi:10.1016/j.tmaid.2020.101578.

[17] H. Lu, "Drug treatment options for the 2019-new coronavirus (2019-nCoV)," Biosci. Trends, 2020, Doi:10.5582/bst.2020.01020.

[18] Z. Xu, C. Peng, Y. Shi, Z. Zhu, K. Mu, and X. Wang, "Nelfinavir was predicted to be a potential inhibitor of 2019-nCov main protease by an integrative approach combining homology modelling , molecular docking and binding free energy calculation," vol. 1201, pp. 0-2, 2020. Doi: 10.1101/2020.01.27.921627

[19] Kapoor, R., Sharma, B. and Kanwar, S.S., 2017. Antiviral phytochemicals: an overview. Biochem. Physiol, 6(2), p.7. Doi: 10.4172/2168-9652.1000220

[20] Naithani, R., Mehta, R.G., Shukla, D., Chandersekera, S.N. and Moriarty, R.M., 2010. Antiviral activity of phytochemicals: a current perspective. In Dietary Components and Immune Function (pp. 421-468). Humana Press, Totowa, NJ. Doi: 10.1007/978-1-60761-061-8_24

[21] Liu, X.; Zhang, B.; Jin, Z.; Yang, H.; Rao, Z. The crystal structure of 2019-nCoV main protease in complex with an inhibitor N3. PDB ID 6LU7 dated 12-Feb-2020.

[22] Jin, Z. et al. (2020). Structure-based drug design, virtual screening and high-throughput screening rapidly identify antiviral leads targeting COVID-19. Doi:10.1101/2020.02.26.964882 
[23] Burley, S. K., Berman, H. M., Kleywegt, G. J., Markley, J. L., Nakamura, H. \& Velankar, S. (2017). Protein Data Bank (PDB): The Single Global Macromolecular Structure Archive. Methods in molecular biology (Clifton, N.J.), 1607, 627-641.

[24] D.S. BIOVIA, Discovery Studio Modeling Environment, Release 2017, San Diego, Dassault Systèmes. (2016).

[25] Parida, M.M., Upadhyay, C., Pandya, G. and Jana, A.M., 2002. Inhibitory potential of neem (Azadirachta indica Juss) leaves on dengue virus type-2 replication. Journal of ethnopharmacology, 79(2), pp.273-278. Doi: 10.1016/S0378-8741(01)00395-6

[26] Ahmad, A., Javed, M.R., Rao, A.Q. and Husnain, T., 2016. Designing and screening of universal drug from neem (Azadirachta indica) and standard drug chemicals against influenza virus nucleoprotein. BMC complementary and alternative medicine, 16(1), p.519. Doi: 10.1186/s12906-016-1469-2

[27] Sahoo, M., Jena, L., Rath, S.N. and Kumar, S., 2016. Identification of suitable natural inhibitor against influenza A (H1N1) neuraminidase protein by molecular docking. Genomics \& informatics, 14(3), p.96. Doi: 10.5808/GI.2016.14.3.96

[28] Shimizu, M., Ito, T., Terashima, S., Hayashi, T., Arisawa, M., Morita, N., Kurokawa, S., Ito, K. and Hashimoto, Y., 1984. Inhibition of lens aldose reductase by flavonoids. Phytochemistry, 23(9), pp.1885-1888. Doi: 10.1016/S0031-9422(00)84935-3

[29] Gaudry, A., Bos, S., Viranaicken, W., Roche, M., Krejbich-Trotot, P., Gadea, G., Desprès, P. and El-Kalamouni, C., 2018. The flavonoid isoquercitrin precludes initiation of Zika virus infection in human cells. International journal of molecular sciences, 19(4), p.1093.Doi: 10.3390/ijms 19041093.

[30] K Parvez, M., H Arbab, A., S Al-Dosari, M. and J Al-Rehaily, A., 2016. Antiviral natural products against chronic hepatitis B: recent developments. Current pharmaceutical design, 22(3), pp.286-293. Doi:10.2174/1381612822666151112152733

[31] Moghaddam, E., Teoh, B.T., Sam, S.S., Lani, R., Hassandarvish, P., Chik, Z., Yueh, A., Abubakar, S. and Zandi, K., 2014. Baicalin, a metabolite of baicalein with antiviral activity against dengue virus. Scientific reports, 4, p.5452. Doi: 10.1038/srep05452

[32] Gosse, B., Gnabre, J., Bates, R.B., Dicus, C.W., Nakkiew, P. and Huang, R.C.C., 2002. Antiviral Saponins from Tieghemella h eckelii. Journal of natural products, 65(12), pp.19421944. Doi: 10.1021/np020165g

[33] Esposito, F., Carli, I., Del Vecchio, C., Xu, L., Corona, A., Grandi, N., Piano, D., Maccioni, E., Distinto, S., Parolin, C. and Tramontano, E., 2016. Sennoside A, derived from the traditional chinese medicine plant Rheum L., is a new dual HIV-1 inhibitor effective on HIV-1 replication. Phytomedicine, 23(12), pp.1383-1391. Doi: 10.1016/j.phymed.2016.08.001

[34] Huang, C.T., Hung, C.Y., Hseih, Y.C., Chang, C.S., Velu, A.B., He, Y.C., Huang, Y.L., Chen, T.A., Chen, T.C., Lin, C.Y. and Lin, Y.C., 2019. Effect of aloin on viral neuraminidase and 
hemagglutinin-specific T cell immunity in acute influenza. Phytomedicine, 64, p.152904. Doi: 10.1016/j.phymed.2019.152904

[35] Choi, K.C., Jung, M.G., Lee, Y.H., Yoon, J.C., Kwon, S.H., Kang, H.B., Kim, M.J., Cha, J.H., Kim, Y.J., Jun, W.J. and Lee, J.M., 2009. Epigallocatechin-3-gallate, a histone acetyltransferase inhibitor, inhibits EBV-induced B lymphocyte transformation via suppression of RelA acetylation. Cancer research, 69(2), pp.583-592. Doi: 10.1158/0008-5472

[36] Sharma, N., Mishra, K.P., Chanda, S., Bhardwaj, V., Tanwar, H., Ganju, L., Kumar, B. and Singh, S.B., 2019. Evaluation of anti-dengue activity of Carica papaya aqueous leaf extract and its role in platelet augmentation. Archives of virology, 164(4), pp.1095-1110. Doi: 10.1007/s00705019-04179-z.

[37] Houghton, P.J., Woldemariam, T.Z., Watanabe, Y. and Yates, M., 1999. Activity against Mycobacterium tuberculosis of alkaloid constituents of Angostura bark, Galipea officinalis. Planta medica, 65(03), pp.250-254. Doi: 10.1055/s-1999-13988

[38] He, M., Min, J.W., Kong, W.L., He, X.H., Li, J.X. and Peng, B.W., 2016. A review on the pharmacological effects of vitexin and isovitexin. Fitoterapia, 115, pp.74-85. Doi: 10.1016/j.fitote.2016.09.011

[39] Fazal, F., Mane, P.P., Rai, M.P., Thilakchand, K.R., Bhat, H.P., Kamble, P.S., Palatty, P.L. and Baliga, M.S., 2014. The phytochemistry, traditional uses and pharmacology of Piper Betel. linn (Betel Leaf): A pan-asiatic medicinal plant. Chinese journal of integrative medicine, pp.1-11. Doi: $10.1007 / \mathrm{s} 11655-013-1334-1$

[40] Salehi, B., Venditti, A., Sharifi-Rad, M., Kręgiel, D., Sharifi-Rad, J., Durazzo, A., Lucarini, M., Santini, A., Souto, E.B., Novellino, E. and Antolak, H., 2019. The therapeutic potential of apigenin. International journal of molecular sciences, 20(6), p.1305. Doi: 10.3390/ijms20061305

[41] Mitrocotsa, D., Mitaku, S., Axarlis, S., Harvala, C. and Malamas, M., 2000. Evaluation of the antiviral activity of kaempferol and its glycosides against human cytomegalovirus. Planta medica, 66(04), pp.377-379.

[42] Ding, Y., Cao, Z., Cao, L., Ding, G., Wang, Z. and Xiao, W., 2017. Antiviral activity of chlorogenic acid against influenza A (H1N1/H3N2) virus and its inhibition of neuraminidase. Scientific reports, 7, p.45723. Doi: 10.1038/srep45723

[43] Dallakyan, S. and Olson, A.J., 2015. Small-molecule library screening by docking with PyRx. In Chemical biology (pp. 243-250). Humana Press, New York, NY. Doi: 10.1007/978-14939-2269-7_19

[44] Y. Li, J. Zhang, N. Wang, et al., Therapeutic drugs targeting 2019-nCoV main protease by high-throughput screening, bioRxiv (2020), Doi: 10.1101/2020.01.28.922922.

[45] RA L, MB S. LigPlot+: multiple ligand-protein interaction diagrams for drug discovery. J Chem Inf Model 2011;51:2778-86. 
[46] Daina A, Michielin O, Zoete V. SwissADME: A free web tool to evaluate pharmacokinetics, drug-likeness and medicinal chemistry friendliness of small molecules. Sci Rep 2017;7:1-13. Doi:10.1038/srep42717.

[47] Daina A, Zoete V. A BOILED-Egg To Predict Gastrointestinal Absorption and Brain Penetration of Small Molecules. ChemMedChem 2016:1117-21. Doi:10.1002/cmdc.201600182.

[48] Anand, K., Ziebuhr, J., Wadhwani, P., Mesters, J.R. and Hilgenfeld, R., 2003. Coronavirus main proteinase (3CLpro) structure: basis for design of anti-SARS drugs. Science, 300(5626), pp.1763-1767. Doi: 10.1126/science. 1085658

[49] Tchesnokov, E.P., Feng, J.Y., Porter, D.P. and Götte, M., 2019. Mechanism of inhibition of Ebola virus RNA-dependent RNA polymerase by remdesivir. Viruses, 11(4), p.326. Doi: 10.3390/v11040326

[50] van der Leur, M.R., Burger, D.M., la Porte, C.J. and Koopmans, P.P., 2006. A retrospective TDM database analysis of interpatient variability in the pharmacokinetics of lopinavir in HIVinfected adults. Therapeutic drug monitoring, 28(5), pp.650-653. Doi: 10.1097/01.ftd.0000245681.12092.d6 